\title{
Role of the hippocampus in contextual memory after classical aversive conditioning in pigeons (C. livia)
}

\section{A.A. Schenka, \\ L.L. Melo and \\ E.A.M. Ferrari}

\author{
Laboratório de Sistemas Neurais e Comportamento, \\ Departamento de Biofísica e Fisiologia, Instituto de Biologia, \\ Universidade Estadual de Campinas, Campinas, SP, Brasil
}

\section{Correspondence \\ E.A.M. Ferrari \\ Departamento de Fisiologia e \\ Biofísica, IB, UNICAMP \\ Cidade Universitária \\ 13083-970 Campinas, SP \\ Brasil \\ Fax: + 55-19-289-3124 \\ E-mail: elenice@ obelix.unicamp.br \\ Research supported by FAPESP, CNPq and FAEP-UNICAMP. F. Reis and A.A. Schenka were the recipients of fellowships from FAPESP for undergraduate research.}

Received January 25, 1999 Accepted June 9, 1999

\begin{abstract}
We investigated the effects of hippocampal lesions with ibotenic acid (IBO) on the memory of the sound-context-shock association during reexposure to the conditioning context. Twenty-nine adult pigeons were assigned to a non-lesioned control group ( $\mathrm{CG}, \mathrm{N}=7$ ), a shamlesioned group ( $\mathrm{SG}, \mathrm{N}=7$ ), a hippocampus-lesioned experimental group (EG, $\mathrm{N}=7$ ), and to an unpaired nonlesioned group (tone-alone exposure) ( $\mathrm{NG}, \mathrm{N}=8$ ). All pigeons were submitted to a 20 -min session in the conditioning chamber with three associations of sound $(1000 \mathrm{~Hz}, 85 \mathrm{~dB}, 1 \mathrm{~s})$ and shock (10 mA, $1 \mathrm{~s})$. Experimental and sham lesions were performed $24 \mathrm{~h}$ later (EG and SG) when EG birds received three bilateral injections (anteroposterior (A), 4.5, 5.25 and 7.0) of IBO $(1 \mu \mathrm{l}$ and $1 \mu \mathrm{g} / \mu \mathrm{l})$ and SG received one bilateral injection (A, 5.25) of PBS. The animals were reexposed to the training context 5 days after the lesion. Behavior was videotaped for $20 \mathrm{~min}$ and analyzed at 30-s intervals. A significantly higher percent rating of immobility was observed for CG (median, 95.1; range, 79.2 to 100.0) and SG (median, 90.0; range, 69.6 to 95.0) compared to EG (median, 11.62; range, 3.83 to 50.1 ) and $\mathrm{NG}$ (median, 7.33; range, 6.2 to 28.1) $(\mathrm{P}<0.001)$ in the training context. These results suggest impairment of contextual fear in birds who received lesions one day after conditioning and a role for the hippocampus in the modulation of emotional aversive memories in pigeons.
\end{abstract}

Classical conditioning provides an association between a stimulus (CS), such as a tone, and an unconditioned stimulus (US), such as a shock. As a result, the CS acquires control over responses elicited by the shock. In these situations the US is also associated with the environment where the conditioning occurs, commonly referred to as the training context $(1,2)$. Several studies $(3-8)$ have emphasized the essential role of the mammalian hippocampus concerning spatial and contextual aspects of learning and memory. Recent studies with mammals have shown that the hippocampus plays a role in aversive conditioning $(3,5,9,10)$.

The avian hippocampus is also known to play a role in spatial memory, including memory-based retrieval of food caches by food- 
storing birds (11-13). Experience of storing and retrieving food is associated with hippocampal enlargement and increased neuronal number (13). The hippocampal region of pigeons, which includes a medial hippocampus and a dorsomedial parahippocampus, as in mammals, is organized into three layers involving different cell groups and is located close to the lateral ventricle $(11,14)$.

The aim of the present study was to investigate the role of the avian hippocampus in contextual learning and memory in an aversive classical tone-shock conditioning situation involving hippocampal lesion with ibotenic acid.

Twenty-nine adult male feral pigeons (derived from Columba livia), averaging 350.0 $\mathrm{g}$ in weight and $20 \mathrm{~cm}$ in height, were housed in individual cages with free acess to food and water, on a 12:12-h light-dark cycle. The birds were divided into four groups: experimental group animals (EG, $\mathrm{N}=7$ ) were submitted to hippocampal lesion with ibotenic acid one day after sound-shock association; sham group animals ( $\mathrm{SG}, \mathrm{N}=7$ ) received an injection of PBS solution in the hippocampus one day after sound-shock association; unoperated control group animals $(\mathrm{CG}, \mathrm{N}=7$ ) were submitted to the same protocol as used for EG and SG animals, except for surgery, and an unpaired nonlesioned group ( $\mathrm{NG}, \mathrm{N}=8$ ) received only tones, instead of tone-shock pairings. A chamber with wooden walls ( $40 \times 40 \times 50 \mathrm{~cm}$ ) was used for exposure to stimuli and for testing. This chamber had its internal walls and floor covered with aluminum plates and a front door with a one-way mirror, and was illuminated by a yellow incandescent lamp $(20 \mathrm{~W})$. A Berger AS-109 sound stimulator (Brasilia, DF, Brazil), located in an adjacent control room, was used for sound presentation. A Novik $80 \mathrm{~W}$ loudspeaker was located on the left wall of the chamber, $20 \mathrm{~cm}$ above the floor. A VHS Panasonic system for video recording (AG-450 camera and AG 1960 tape recorder) connected to a monitor was used to record the sessions. Electric shocks were delivered via two stainless steel electrodes chronically implanted in the pigeon's pubic arch.

The aversive conditioning consisted of a 20-min session with three tone-shock associations. In each association the sound (1000 $\mathrm{Hz}, 85 \mathrm{~dB}, 1 \mathrm{~s}$ ) offset was contiguous to the shock $(10 \mathrm{~mA}, 1 \mathrm{~s})$ onset. Twenty-four hours later, the birds were anesthetized with xylasine and ketamine $(0.1 \mathrm{mg} / \mathrm{kg}, 1: 1)$ and placed in a stereotaxic instrument (David Kopf, model 1204, Tujunga, CA, USA, with a Revzin adaptor for pigeons). The lesion was performed with ibotenic acid $(1 \mathrm{mg} / 0.1$ $\mathrm{ml}$ in 0.1 M PBS, pH 7.4). A 10- $\mu$ l Hamilton syringe attached to a cannula was used for bilateral injection into the following sites: anteroposterior (A), 4.5, 5.25, 7.0; ventral (V), 2.5; lateral (L), 0.3 (15). Sham-lesioned birds received a bilateral injection of $1 \mu \mathrm{l}$ PBS into the same sites as follows: A, 5.2; V, 2.5; L, 0.3 (15). Five days later, all animals were reexposed to the training context in which sound-shock pairing or sound alone presentation occurred in a 20-min session. Behavior was videotaped throughout the session. The occurrence of immobility, preexploratory and exploratory responses, locomotion, preening and isolated movements was recorded every $30 \mathrm{~s}$ by two independent observers. The percent value of each response category was calculated relative to the total behaviors displayed by the animal during the test session. Reliability was checked in random sessions and the minimum acceptable reliability index was $80 \%$ agreement between the two observers (16). Differences between groups were evaluated by Kruskal-Wallis analysis of variance for nonparametric data, followed by post-hoc Ztest comparisons.

Following the behavioral tests, the pigeons were sacrificed and their brains were placed in formaldehyde-saline solution. The brains were then sectioned along the frontal plane at $10 \mu \mathrm{m}$ for staining according to the 
Klüver-Barrera technique.

The tone-shock pairings were effective in producing conditioning to the context (Figure 1) as indicated by a significantly different percent rating of immobility for CG (median 95.1, range 79.2 to 100.0 ) and SG (median 90.0, range 69.6 to 95.0 ) in comparison to NG (median 7.33, range 6.2 to 28.1 ) and EG (median 11.62, range 3.83 to 50.1). Analysis of the percent values of exploratory behaviors showed that EG (median 27.9, range 13.7 to 55.8) and NG (median 61.4, range 54.8 to 71.9 ) animals presented a higher frequency of exploration which was different from that observed in SG (median 0.0, range 0.0 to 15.3) and CG (median 2.6, range 0.0 to 7.9$)$. The nonparametric KruskalWallis analysis revealed a significant effect of group $(\mathrm{H}[3,25]=16.64, \mathrm{P}<0.001)$. Posthoc Z-tests for pairwise comparisons showed that EG was significantly different from CG and SG $(\mathrm{P}<0.05)$ but not from NG ( $\mathrm{P}>0.05)$.

Figure 2 is a schematic representation of the pigeon's brain in frontal sections (15). The localization of the lesion was considered to be representative for all the experimental birds. Morphological analysis indicated that the lesioned areas were characterized by severely damaged neuronal bodies.

The present data showed long lasting immobility when SG and CG animals were reexposed to the context of aversive classical conditioning. These birds presented a characteristic crouching posture with a) total flexion of the legs and the body in contact with the floor or $b$ ) partial flexion of the legs, wide separation between feet/legs and the pectoral region in close contact with one of the walls. The eyes remained widely opened and respiration was accelerated. Such posture, associated with the absence of other observable behaviors, was considered to express tense immobility, characterizing freezing behavior in pigeons $(17,18)$.

The fact that immobility/freezing responses prevailed over other classes of be- havior in SG and CG birds may be interpreted as evidence of contextual memory in the pigeons which were not submitted to experimental lesion. These responses may represent a behavioral counterpart of conditioned fear since they are elicited by the contextual stimuli which are identified as potentially dangerous $(17,18)$. This set of stimuli present in the situation of soundshock pairing was associated with the shock and acquired an aversive function (10,1820). These assumptions may be supported by the observation that birds exposed to sound-alone presentations predominantly

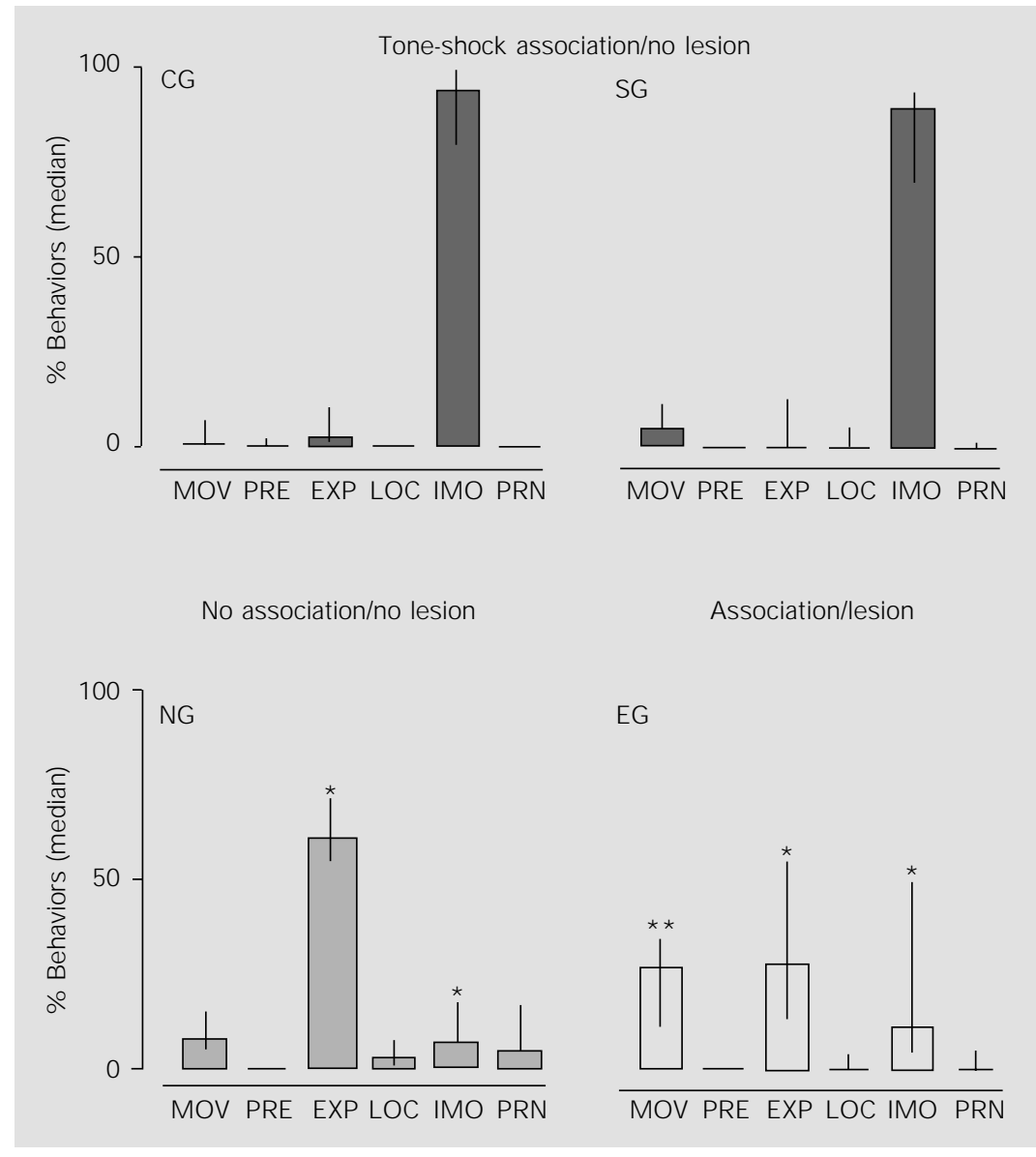

Figure 1 - Effects of tone-shock association and hippocampal lesion on behavioral categories observed during context test session. Data are reported as median of percent values (interquartile range) of isolated movements (MOV), pre-exploratory movements (PRE), exploratory movements (EXP), locomotion (LOC), immobility (IMO) and preening (PRN) relative to the total of behaviors. NG: Naive group; CG: control group; SG: sham group; EG: experimental group. *P<0.05 compared to CG and SG; **P<0.05 compared to CG (KruskalWallis test, followed by the multiple comparisons Z-test). 
Figure 2 - Schematic representations of frontal sections of the pigeon's brain according to Karten and Hodos (15). The minimal (darker areas) and the maximal extent of the lesions in hippocampal and parahippocampal regions are indicated. $\mathrm{E}=\mathrm{Ecto}-$ striatum; $\mathrm{N}=$ neostriatum; $\mathrm{PA}=$ paleostriatum augmentatum; PP $=$ paleostriatum primitivum; $\mathrm{LHy}$ $=$ nucleus lateralis hypothalami.

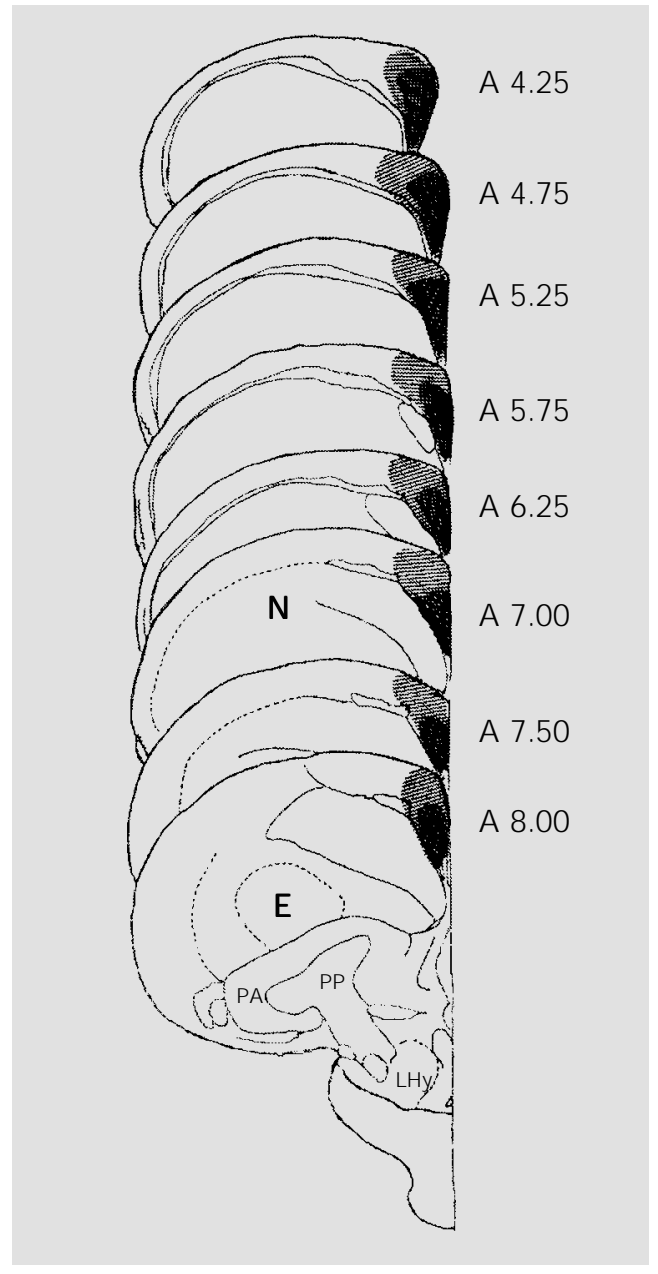

showed behaviors associated with relaxed postures. These results indicate that the sound-context association, per se, had no aversive properties.
Like the NG group, the EG animals showed a higher rate of exploration and relaxed postures. These data indicate that, in contrast to the SG and CG groups, the context of sound-shock pairing had a lower aversive value for the hippocampus-lesioned birds. The lower occurrence of tense immobility/freezing in EG birds may indicate an impairment of the consolidation of the contextual memory after classical aversive conditioning. The present data are relevant to the recent reports concerning the role of the mammalian hippocampus in aversive classical conditioning $(4,5,7,9,10)$. Indeed, in addition to describing the pigeons' freezing behavior in response to a context of aversive conditioning, our data show contextual memory deficits when the hippocampus is lesioned $24 \mathrm{~h}$ after conditioning. Therefore, the present data indicate that the role of the avian hippocampus in contextual aversive memory is consistent with that already described for mammals.

\section{Acknowledgments}

We are indebted to V.S. Valentinuzzi for statistical assistance and to M.C.C.G. Marcondes and F. Langone for assistance with the scanning procedures. The authors also thank W.L. Gomes for animal care and technical support.

\section{References}

1. Landeira-Femandez J (1996). Context and Pavlovian conditioning. Brazilian J ournal of Medical and Biological Research, 29: 149-173.

2. Rescorla RA \& Wagner AR (1972). A theory of Pavlovian conditioning: variations in the effectiveness of reinforcement and non-reinforcement. In: Black AH \& Prokasy WF (Editors), Classical Conditioning II: Current Research and Theory. Appleton-Century-Crofts, New York.

3. Eichenbaum H \& Otto T (1992). The hippocampus - What does it do? Behavioral and Neural Biology, 57: 2-36.

4. Kim J J \& Fanselow MS (1992). Modality- specific retrograde amnesia of fear. Science, 256: 675-677.

5. Maren S \& Fanselow MS (1995). Synaptic plasticity in the basolateral amygdala induced by hippocampal formation stimulation in vivo. J ournal of Neuroscience, 15: 7548-7564.

6. Mayes R (1995). Memory and amnesia. Behavioural Brain Research, 66: 29-36.

7. Schmajuk NA \& DiCarlo JJ (1992). Stimulus configuration, classical conditioning and hippocampal function. Psychological Review, 99: 268-305.

8. Scoville WB \& Milner B (1957). Loss of recent memory after bilateral hippocam- pal lesions. J oumal of Neurology, Neurosurgery and Psychiatry, 20: 11-12.

9. Maren $\mathrm{S}$, Aharavov $\mathrm{G} \&$ Fanselow MS (1997). Neurotoxic lesions of the dorsal hippocampus and Pavlovian fear conditioning in rats. Behavioural Brain Research, 88: 261-274.

10. Winocur G (1997). Hippocampal lesions alter conditioning to conditional and contextual stimuli. Behavioural Brain Research, 88: 219-229.

11. Bingman VP, Bagnoli $P$, loalè $P \&$ Casini $G$ (1989). Behavioral and anatomical studies of the avian hippocampus. Neurology and Neurobiology, 52: 379-394. 
12. Healy SD, Gwinner E \& Krebs J R (1996). Hippocampal volume in migratory and non migratory warblers: effects of age and experience. Behavioural Brain Research, 81: 61-68.

13. Patel SJ , Clayton NS \& Krebs JR (1997). Spatial learning induces neurogenesis in the avian brain. Behavioural Brain Research, 89: 115-128.

14. Veeman CL, Wild J M \& Reiner A (1995). Organization of the avian "corticostriatal" projection system: a retrograde and anterograde pathway tracing study in pigeons (C. livia). J ournal of Comparative Neurology, 354: 87-126.
15. Karten HJ \& Hodos W (1967). A Stereotaxic Atlas of the Brain of the Pigeon (Columba livia). J ohns Hopkins University Press, Baltimore.

16. Ferrari EAM, Faleiros L, Cerutti SM \& Oliveira AM (1999). The functional value of sound and exploratory behaviour in detelencephalated pigeons. Behavioural Brain Research, 101: 93-103.

17. Bamett SA \& Cowan PE (1976). Activity, exploration, curiosity and fear: an ethological study. Interdisciplinary Science Reviews, 1: 43-62.

18. Graeff FG (1994). Neuroanatomy and neurotransmitter regulation of defensive be- haviors and related emotions in mammals. Brazilian J ournal of Medical and Biological Research, 27: 811-829.

19. Good M \& Macphail EM (1994). Hippocampal lesions in pigeons (Columba livia) disrupt reinforced preexposure but not overshadowing or blocking. Quarterly J oumal of Experimental Psychology, 47B: 263-291.

20. Weinberger NM (1995). Retuning the brain by fear conditioning. In: Gazzaniga MS (Editor), The Cognitive Neurosciences. MIT Press, Cambridge, MA. 